\title{
Dual-gate self-aligned a-InGaZnO transistor model for flexible circuit applications
}

\author{
Florian De Roose \\ imec, large area electronics \\ KU Leuven, ESAT department \\ Leuven, Belgium \\ florian.deroose@imec.be \\ Wim Dehaene \\ KU Leuven, MICAS department \\ imec, large area electronics \\ Leuven, Belgium
}

\author{
Hikmet Çeliker \\ imec, large area electronics \\ KU Leuven, ESAT department \\ Leuven, Belgium
}

Kris Myny

imec, large area electronics

Leuven, Belgium

\author{
Jan Genoe \\ imec, large area electronics \\ KU Leuven, ESAT department \\ Leuven, Belgium
}

\begin{abstract}
This work elaborates on an amorphous Indium-Gallium-Zinc Oxide thin-film transistor model for a dual-gate self-aligned transistor configuration, enabling the design and realization of complex integrated circuits. The model originates from a mobility-enhanced transistor behavior model, whereby the additional backgate impacts key parameters, such as threshold voltage, mobility and subthreshold slope. The model has been validated for the full design flow and compared to measurement results, from single transistors, to inverters, ring oscillators and RFID transponder chips.
\end{abstract}

Keywords-InGaZnO, mobility-enhancement, dual-gate, modeling, integrated circuits, RFID transponder chips

\section{INTRODUCTION}

Thin-film transistor (TFT) technologies are attractive for item-level Internet-of-Things applications requiring ultra-thin, flexible integrated circuits operating at low power to enable seamless integration into objects. Metal-oxide thin-film transistors (TFTs), amongst others IndiumGallium-Zinc-Oxide or InGaZnO are ideal candidates for these applications, resulting in n-type transistors with an electron mobility around $10-20 \mathrm{~cm}^{2} / \mathrm{Vs}$ [1]. Recent developments in this field have demonstrated phone-detectable near field communication (NFC) tags compatible to ISO14443 standard [2]. The realization of such demonstrator requires, besides maturity in technology, also increased maturity in the design flow and its simulation models. The design flow was based on a standard cell place-and-route for the core of the chip and a manual placement for the partition that requires high-speed operation to avoid substantial parasitic overlap due to automatic routing algorithms. The focus of this work will be the InGaZnO transistor model enabling SPICE predictions of the circuits' behavior.

The quality of the prediction of the currents and voltages in between the terminals of the TFT will impact directly the accuracy of the DC simulation parameters. Transient analysis of the circuit requires to additionally model the capacitances of the TFT and parasitic resistances and capacitances of the circuit. A dual-gate transistor has four terminals: gate (G), source (S), drain (D) and backgate (BG). The cross-section of a dual-gate TFT and the different currents and voltages are shown in Fig. 1. Depending on technology, the gate and back-gate terminal may be swapped and may differ in symmetry.

\section{MoBILITY-ENHANCEMENT MODEL}

In the simplest case of a single gate device, the saturation current of a transistor can be described by a quadratic equation according to Shichman and Hodges [3]:

$$
I_{D}=\frac{\mu C_{o x}}{2} \frac{W}{L}\left(V_{G S}-V_{T}\right)^{2}
$$

where $\mu$ defines the mobility and $\mathrm{C}_{\mathrm{ox}}$ the gate oxide capacitance. $\mathrm{W}$ and $\mathrm{L}$ are the electrical width and length, and $\mathrm{V}_{\mathrm{T}}$ is the threshold voltage. Similar as many thin-film semiconductors [4], [5], [6], InGaZnO shows a larger mobility for larger values of $V_{G s}$. The apparent mobility enhancement effect can be described by a less than linear relation with respect to $\mathrm{V}_{\mathrm{GS}}$ :

$$
\mu=\mu_{0}\left(V_{G S}-V_{T}\right)^{\gamma}
$$

Here, $\gamma$ is the mobility enhancement factor, $\mu_{0}$ is defined as the mobility at $V_{G S}-V_{T}=0$, so it does not represent the maximum mobility of the device anymore. The saturation current for a single-gate InGaZnO transistor can therefore be described as [5]:

$$
I_{D}=\frac{\mu_{0} C_{o x}}{2} \frac{W}{L}\left(V_{G S}-V_{T}\right)^{2+\gamma}
$$

Thin-film devices are intrinsically symmetrical due to the lack of bulk contact, so the transition from saturation to linear regime can best be described by an equation that represents this symmetry. Therefore, the subtraction of two saturation currents can be used: the forward and reverse current. The forward current is determined by $V_{S}$, while the reverse current is determined by $V_{D}$. The total current then is [5]:

$$
I_{D}=\frac{\mu_{0} C_{o x}}{2} \frac{W}{L}\left[\left(V_{G}-V_{S}-V_{T}\right)^{2+\gamma}-\left(V_{G}-V_{D}-V_{T}\right)^{2+\gamma}\right]
$$

The extension in the subthreshold region for single gate modeling can be found in [5].

\section{INTRODUCTION OF THE BACKGATE}

The technology comprises of a thin-film transistor having an individual controllable backgate per transistor, similar as FD-SOI technologies. This feature is beneficial for TFT-based applications, enabling to tune the threshold voltage of each transistor [7]. Typically, only the $\mathrm{V}_{\mathrm{T}}-$ shift is modeled with the backgate. This limits the prediction accuracy for our TFT measurements, shown in Fig. 2.

A better approximation can be made by extracting the mobility-enhancement model for every value of $\mathrm{V}_{\mathrm{BG}}$ and evaluate the change in both mobility and $\gamma$ depending on the $\mathrm{V}_{\mathrm{BG}}$. The values for $\mathrm{V}_{\mathrm{T}}, \gamma$ and subthreshold slope (SS) can be estimated by a linear function. $\mu_{0}$ can be estimated as a quadratic function.

$$
V_{T}=V_{T O}-\zeta V_{B G}
$$




$$
\begin{gathered}
\gamma=\gamma_{0}+\gamma_{1} V_{B G} \\
\mu_{0}=\mu_{00}+\mu_{01} V_{B G}+\mu_{02} V_{B G}^{2} \\
S S=S S_{0}+S S_{1} V_{B G}
\end{gathered}
$$

Here, $\zeta$ is the ratio of capacitive coupling between front and backgate, while $V_{T 0}, \mu_{00}, \gamma_{0}$ and $S S_{0}$ are the threshold voltage, mobility, mobility enhancement factor and subthreshold slope for $V_{B G}=0$. The other parameters have no physical meaning and are purely there to improve the quality of the prediction. Fig. 3 shows the fit of all transistor parameters according to these equations. It can be observed that the equations fit very well. Fig. 4 plots the transistor measurements after applying different backgate voltages and compares these to the simulated model after fitting the parameters. A good fit is observed for the on-behavior of the TFT.

In the transition from linear to saturation, the approach where $\mathrm{V}_{\mathrm{T}}$ is only dependent on $V_{B G}$ is not sufficient. The model above assumes that a change in backgate influences the parameters such as mobility for the forward and backward current identically, as e.g. the mobility is shifted by a fixed value. This is referred to as the global backgate model.

Fig. 5 depicts the output characteristic for the global backgate model and compares it to the measurements. Saturation occurs much sooner on the VDS axis than predicted by the model. The solution is to adopt a local backgate model. Since the forward current is caused by the overdrive voltage at the source and the reverse current is caused by the overdrive voltage at the drain, also the expressions for the shift in mobility need to be applied depending on the local (source or drain) backgate overdrive voltage $\mathrm{V}_{\mathrm{BGX}}$. The shift in $\mathrm{V}_{\mathrm{T}}, \gamma, \mu_{0}$ and $\mathrm{SS}$ then depends on this local backgate overdrive voltage, not the global backgate overdrive voltage:

$$
V_{B G X}=V_{B G}-V_{X}
$$

Whereby $V_{X}$ is $V_{S}$ and $V_{D}$ for the forward and reverse current respectively. Fig. 5 indicates that the local backgate improves the prediction of earlier saturation. Consequently, the saturation voltage $V_{D S s a t}$ must be calculated differently and occurs when the reverse current becomes 0 :

$$
V_{D S s a t}=\frac{V_{G}+\zeta V_{B G}-V_{T 0}}{1+\zeta}
$$

In addition to the earlier onset of saturation, the output resistance of a dual-gate device is improved compared to a single gate device, due to the enhanced electrostatic control of the channel.

The complete DC model for a dual-gate self-aligned TFT can be found by combining the above-explained equations. This incorporates forward-backward mobility-enhancement current equations, subthreshold behavior and the parameter shift caused by the backgate.

$$
\begin{gathered}
I_{D}=\frac{\mu_{0 S} C_{o x}}{2} \frac{W}{L} V_{G S T e}^{2+\gamma_{S}}-\frac{\mu_{0 D} C_{o x}}{2} \frac{W}{L} V_{G D T e}^{2+\gamma_{D}} \\
V_{G X T e}=\left(2+\gamma_{X}\right) S S_{X} \log _{10}\left(1+10^{\wedge}\left(\frac{V_{G}-V_{X}-V_{T X}}{\left(2+\gamma_{X}\right) S S_{X}}\right)\right) \\
V_{T X}=V_{T 0}-\zeta\left(V_{B G}-V_{X}\right) \\
\gamma_{X}=\gamma_{0}+\gamma_{1}\left(V_{B G}-V_{X}\right) \\
\mu_{0 X}=\mu_{00}+\mu_{01}\left(V_{B G}-V_{X}\right)+\mu_{02}\left(V_{B G}-V_{X}\right)^{2} \\
S S_{X}=S S_{0}+S S_{1}\left(V_{B G}-V_{X}\right)
\end{gathered}
$$

\section{FROM TFT tO INTEGRATED CIRCUITS}

A complex digital integrated chip is built from a standard cell library containing a multitude of simple and complex logic gates. One of the key logic blocks is an inverter gate. The self-aligned dual-gate InGaZnO thin-film transistor technology conducts only electrons, leading to the implementation of a dual-gate diode-connected load inverter gate, detailed in Fig. 6. The drive transistor uses its backgate to shift the voltage transfer curve of the inverter with an external backgate voltage, aiming to maximize noise margin and/or speed.

We have measured 8 dual-gate diode-load inverters at two different bias voltages ( $0 \mathrm{~V}$ and $-2.5 \mathrm{~V})$, shown in Fig. 7, fabricated with the selfaligned dual-gate TFT technology. The variability of the curves stems from the presence of transistor parameter variation on the manufactured wafers. The extracted $V_{T}$ standard deviation on a $150 \mathrm{~mm}$ wafer with our lab process is about $250 \mathrm{mV}$. The red solid line in Fig. 7 shows the simulated curve by using the dual-gate model, with a threshold voltage of $-575 \mathrm{mV}$. It is observed that the slope of the voltage transfer curve is predicted well with the described model, indicating that the currents are well matched with the measured data. Standard cell library design will require a relevant amount of Monte Carlo simulations of the logic gates, taking into account the intrinsic parameter variation of the technology. As such, important parameters such as gain, noise margin, speed and power will be predicted more accurate.

Fig. 8 shows the extracted gain, noise margin and DC power of the inverter gate for the measurements and the model at $0 \mathrm{~V}$ and $-2.5 \mathrm{~V} \mathrm{~V}_{\mathrm{BG}}$. It is observed that the simulated inverter gate predicts the DC parameters accurately, with an error less than $4 \%$.

Another key parameter, next to robustness and gain, is the stage delay of a logic gate. In order to validate this, we have realized 33-stage ring oscillators based on the dual-gate diode-load inverter topology. 5 ring oscillators have been characterized at a supply voltage of $5 \mathrm{~V}$, whereby $\mathrm{V}_{\mathrm{BG}}$ is varied between $0 \mathrm{~V},-1.25 \mathrm{~V}$ and $-2.5 \mathrm{~V}$. The average frequency is plotted in Fig. 9. Simulations of the transient behavior is also performed by including a basic gate capacitance behavior and some parasitic overlap capacitors in the layout of the ring oscillator. The ring oscillator frequency of the simulations is observed to be slower than the measurement results. This can have multiple origins, amongst others an overestimation of the parasitic and gate capacitors which requires to be better modeled, or the fact that $\mathrm{V}_{\mathrm{T}}$ may be different for this location on the wafer due to variability. The threshold voltage impacts directly the on-current and off-current, and therefore the stage delay of the inverters.

Finally, many applications for thin-film electronics would benefit from ultra-low power consumption, such as increasing battery life of onetime usable patches or increasing reading distances of thin-film RFID tags. A good prediction of the power consumption will consequently 
lead to more optimized designs with respect to power consumption. The power consumption of the above-mentioned ring oscillators has been measured and compared to the simulations. The simulated results are relatively close to the measurements, mainly due to static power consumption as a key contributor for unipolar logic gates. These logic gates suffer from a constant leakage current present between supply and ground node.

In addition, the power consumption has also been estimated for a 12-bit RFID transponder chip, employing 494 InGaZnO TFTs. The power consumption has also been characterized on the manufactured transponder chip, with a clock frequency of $100 \mathrm{~Hz}$. The comparison between simulation and measured power consumption at $\mathrm{V}_{\mathrm{BG}}$ of $0 \mathrm{~V}$ and $-2.5 \mathrm{~V}$ is plotted in Fig. 10, detailing a good first order prediction of the power consumption for digital integrated circuits. This power consumption combines both the static and dynamic components, whereby the static portion is mostly dominant for these unipolar dual-gate diode-load logic gates.

\section{CONCLUSIONS}

This work elaborates on a transistor model of a dual-gate self-aligned thin-film transistor technology based on InGaZnO as semiconductor. The basic idea stems from a mobility-enhanced transistor behavior model. The addition of a backgate in this technology features an extra parameter for the designer to optimize the transistor behavior for its needs. It is found that a threshold voltage shift by the backgate is not sufficient to explain the behavior of the device: in addition, the backgate voltage influences the subthreshold slope, mobility and mobility enhancement. The simulation model has been validated for inverters, ring oscillators and 12-bit RFID transponder chips, fabricated with these dual-gate self-aligned TFTs. A good match between the measurement results of the DC parameters of the circuits with the simulations using the model was found.

\section{ACKNOWLEDGMENT}

This work has received funding from the European Research Council (ERC) under the European Union's Horizon 2020 research and innovation programme under grant agreement No 716426 (FLICs project). The described work is partially financed through the Flexlines project within the Interreg V-programme Flanders-The Netherlands, a cross-border cooperation programme with financial support from the European Regional Development Fund.

\section{REFERENCES}

[1] K. Nomura et al., "Room-temperature fabrication of transparent flexible thin-film transistors using amorphous oxide semiconductors," Nature, vol. 432, pp. 488-492, Nov. 2004.

[2] K. Myny, "The development of flexible integrated circuits based on thin-film transistors," Nature Electronics, vol. 1, no. 1, pp. 30-39, Jan. 2018.

[3] H. Shichman and D. Hodges, "Modeling and simulation of insulated-gate field-effect transistor switching circuits," IEEE Journal of Solid-State Circuits, vol. 3, no. 3, pp 285-289, Sept. 1968.

[4] M. Shur and M. Hack, "Physics of amorphous silicon based alloy field-effect transistors," Journal of Applied Physics, vol. 55, no. 10, pp 3831-3842, May 1984.

[5] O. Marinov, M. J. Deen, U. Zschieschang, and H. Klauk, "Organic thin-film transistors: Part I—Compact DC modeling," in IEEE Transactions on Electron Device Modeling, vol. 56, no. 12, pp. 2952-2961, Dec. 2009.

[6] K. Abe, H. Kumomi, T. Kamiya, and H. Hosono, "Modeling of transparent amorphous oxide semiconductor thin-film transistors," Proceedings of the IDW 2013, no. 1, pp. 311-314, Dec. 2013.

[7] Spijkman et al, "Dual-gate thin-film transistors, integrated circuits and sensors," Advanced Materials, vol. 23, no. 29, pp. 3231-3242, 2011. 
FIGURES

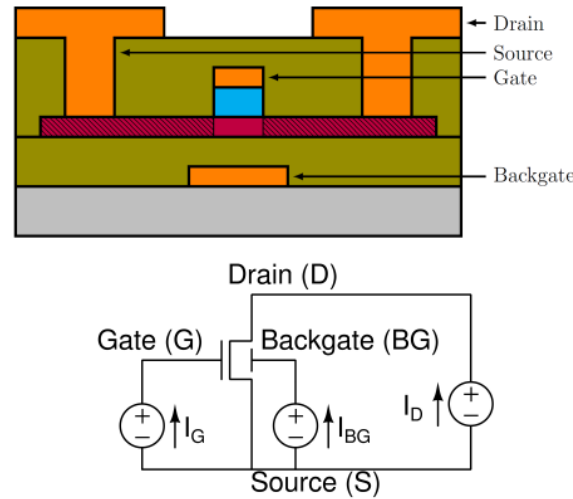

Fig 1: (top) Typical cross-section of a self-aligned dual-gate InGaZnO TFT and (bottom) different currents flowing in a 4-terminal dual-gate TFT
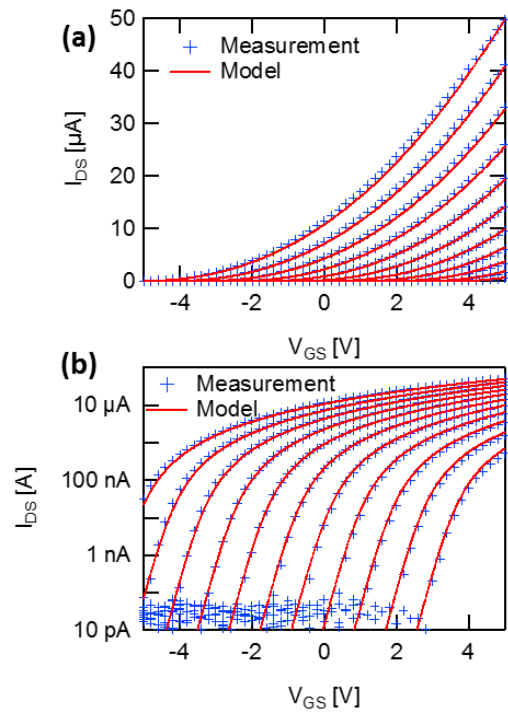

Fig 2: TFT transfer curve comparison between measurement and $\mathrm{V}_{\mathrm{T}}$-shift only model, when $\mathrm{V}_{\mathrm{BG}}$ is varied between $-5 \mathrm{~V}$ and $+5 \mathrm{~V}$
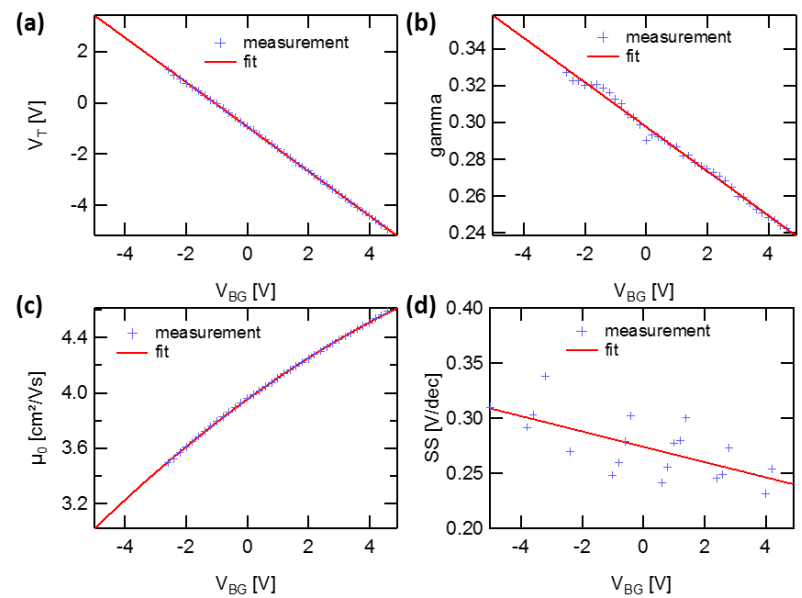

Fig 3: (a) $\mathrm{V}_{\mathrm{T}}$, (b) $\gamma$, (c) mobility and (d) SS model parameters fitted as a function of backgate voltage
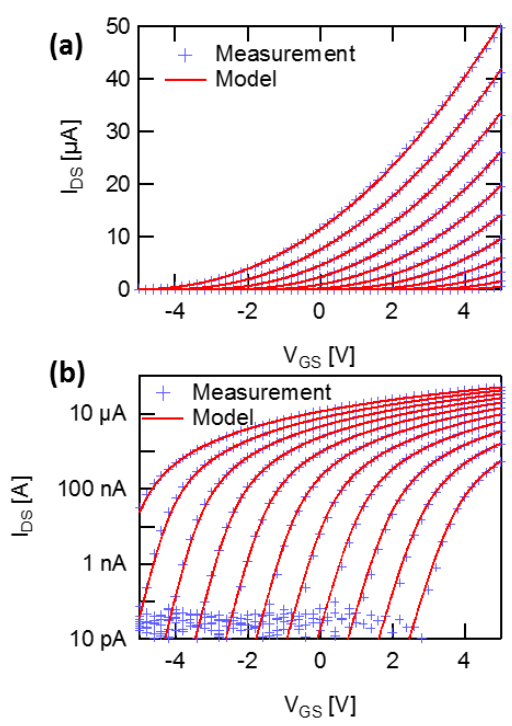

Fig 4: TFT transfer curve comparison between measurements and complete $\mathrm{DC}$ model, when $\mathrm{V}_{\mathrm{BG}}$ is varied between $-5 \mathrm{~V}$ and $+5 \mathrm{~V}$

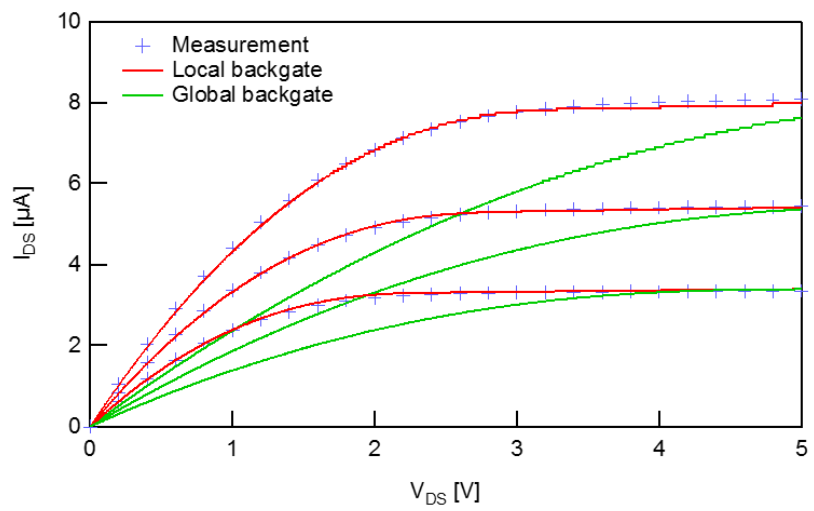

Fig 5: Measurements of a TFT output curve compared to the model with local and global backgate principle

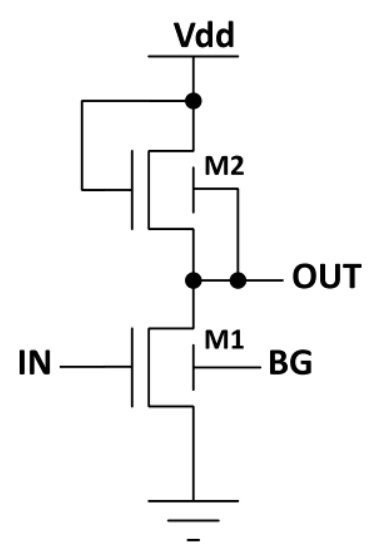

\begin{tabular}{|c|c|}
\hline TFT & $\begin{array}{c}W / L \\
{[\mu \mathrm{m} / \mu \mathrm{m}]}\end{array}$ \\
\hline $\mathrm{M} 1$ & $100 / 5$ \\
\hline $\mathrm{M} 2$ & $10 / 5$ \\
\hline
\end{tabular}

Fig. 6: Schematic of a dual-gate diode-load inverter and the sizes of the TFTs 


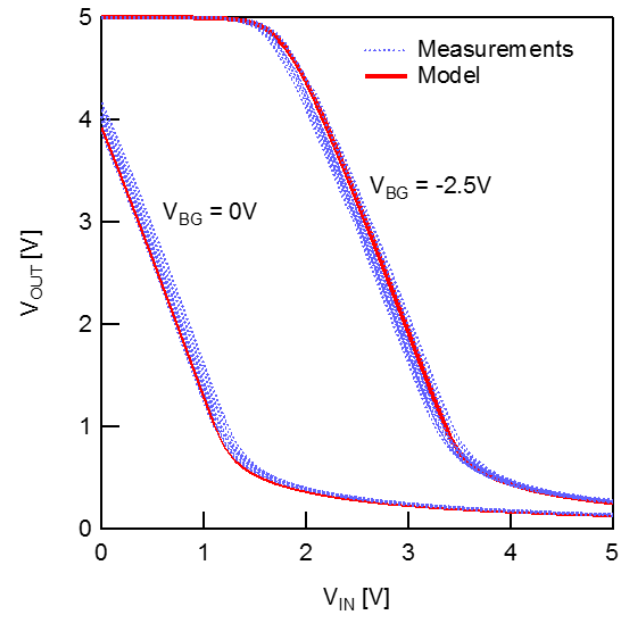

Fig 7: Voltage transfer curves measured for 8 different dual-gate diodeload inverters and compared to the SPICE simulation with the presented model
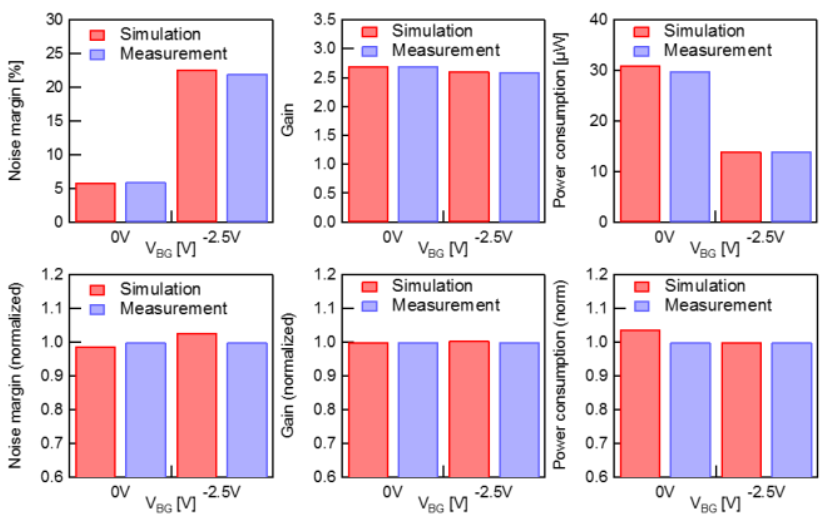

Fig. 8: Comparison of the extracted parameters, gain, noise margin and DC power from the measurements and SPICE simulations of the inverters plotted in Fig. 7 for two different backgate voltages
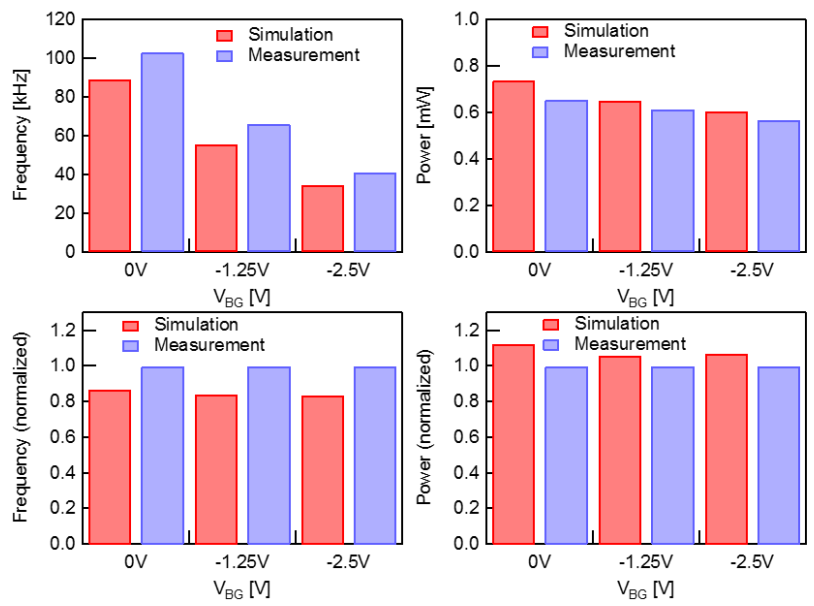

Fig. 9: Comparison of the extracted parameters, frequency and power from the measurements and SPICE simulations of 33-stage ring oscillators

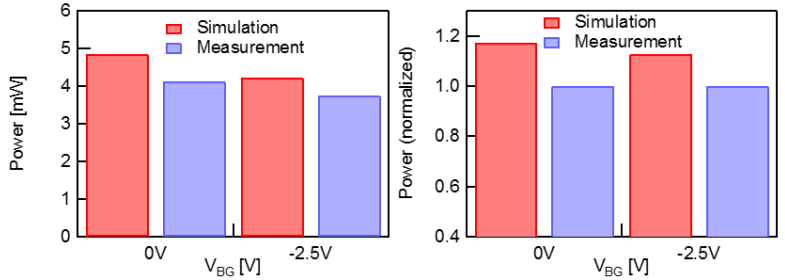

Fig 10: Measurement and SPICE simulation comparison of the power consumption of a 12-bit RFID transponder chip 